\title{
METANÁLISE DE ENSAIOS CLÍNICOS CONTROLADOS ALEATORIZADOS: ASPECTOS QUANTITATIVOS
}

\author{
META-ANALYSIS OF RANDOMIZED CONTROLLED TRIALS: QUANTITATIVE ASPECTS
}

Edson Zangiacomi Martinez

Docente. Departamento de Medicina Social. Faculdade de Medicina de Ribeirão Preto - USP.

CorRespondêncIA: Edson Zangiacomi Martinez, Departamento de Medicina Social, Faculdade de Medicina de Ribeirão Preto-USP. Av. Bandeirantes, 3900 - 14049-900 - Ribeirão Preto - SP. edson@fmrp.usp.br

Martinez EZ. Metanálise de ensaios clínicos controlados aleatorizados: aspectos quantitativos. Medicina (Ribeirão Preto) 2007; 40 (2): 223-35.

RESUMO: A metanálise é uma técnica que combina os resultados de diversos estudos voltados a um conjunto de hipóteses específicas. A metanálise vem sendo amplamente utilizada em estudos médicos, especialmente em revisões sistemáticas de ensaios clínicos aleatorizados. Há, portanto, a necessidade de estudos sobre as ferramentas estatísticas envolvidas. Os resultados dos estudos são combinados por diferentes estratégias. Uma alternativa considera homogeneidade entre os estudos. Modelos de regressão com efeitos fixos são compatíveis com este pressuposto. Por outro lado, se for assumida heterogeneidade entre os estudos, os modelos de efeitos aleatórios são mais adequados. O presente artigo revisa alguns aspectos quantitativos da metanálise.

Descritores: Metanálise. Ensaios Clínicos. Revisão Sistemática. Estatística.

\section{1- INTRODUÇÃO}

Um ensaio clínico é uma forma de experimento planejado que envolve pacientes, designado a elucidar o mais apropriado tratamento para futuros pacientes com uma específica condição ${ }^{1}$. Muitos ensaios buscam testar o efeito de uma droga ou uma intervenção para determinar sua efetividade ou segurança. O planejamento de ensaios clínicos é discutido, por exemplo, por Boissel ${ }^{2}$. Um ensaio clínico controlado aleatorizado envolve ao menos um tratamento sob investigação e um tratamento controle, onde a alocação dos indivíduos aos tratamentos utiliza um processo aleatório (ver, por exemplo, Kendall ${ }^{3}$ ). O tratamento controle envolve uma droga, meio, teste ou procedimento que serve como um padrão para comparação com o(s) tratamento(s) sob investigação. Pode consistir de um placebo, um procedimento simulado, um tratamento convencional, ou mesmo nenhum tipo de tratamento, dependendo do desenho do estudo.

Diz-se que um ensaio clínico é aleatorizado quando os indivíduos elegíveis ao estudo são alocados nos diferentes grupos de tratamentos de maneira casual, segundo, por exemplo, a geração de uma seqüência de números aleatórios em um programa de computador. Em um ensaio aleatorizado, portanto, não há qualquer controle do pesquisador sobre a decisão de destinar um paciente a um ou outro grupo; e nem os pacientes participam desta escolha. $\mathrm{O}$ conceito de alocação aleatória quando comparados diferentes tratamentos, segundo Pocock ${ }^{1}$, recebe grande importância no delineamento de experimentos desde o trabalho pioneiro de Fisher ${ }^{4}$. Os primeiros experimentos aleatorizados foram realizados na agricultura, e suas idéias foram 
posteriormente adaptadas aos outras áreas da pesquisa científica. Os propósitos da aleatorização são: (a) evitar vieses e (b) garantir que os pressupostos exigidos pelos métodos tradicionais de análise estatística sejam respeitados. A importância da aleatorização em ensaios clínicos é discutida por Altman e Bland ${ }^{5}$, que mencionam que o periódico British Medical Journal possui a política de não publicar ensaios que não tenham sido propriamente aleatorizados, a não ser que seus autores apresentem justificativas para isto. Maneiras de garantir que um ensaio clínico seja aleatorizado são discutidas por Robert e Torgerson ${ }^{6}$, Torgerson e Roberts ${ }^{7}$, Altman e Bland ${ }^{8}$ e Pocock ${ }^{1}$.

Uma revisão sistemática ${ }^{9}$ é um tipo de pesquisa planejada para sumarizar estudos médicos, respondendo a uma questão clínica específica. Utiliza métodos apropriados para identificar, selecionar e avaliar criticamente os estudos, e também para analisar os dados destes estudos incluídos na revisão ${ }^{10}$. Segundo Mulrow ${ }^{11}$, profissionais da saúde, pesquisadores e outros responsáveis por políticas de saúde são freqüentemente inundados com quantidades de informação nem sempre manejáveis, o que torna a revisão sistemática uma maneira eficiente de integrar a informação existente, gerando dados que auxiliem a tomada de decisões. Em uma revisão sistemática, os dados dos diferentes estudos podem ser quantitativamente combinados por métodos estatísticos. Estes métodos são chamados de técnicas de metanálise ${ }^{12}$.

Uma etapa importante de uma revisão sistemática consiste em classificar os estudos conforme sua qualidade, selecionando aqueles que irão compor a metanálise. Buscando atenuar a subjetividade desta classificação, alguns sistemas foram propostos, como o Maastricht ${ }^{13}$, Jadad ${ }^{14}$ e Delphi ${ }^{15}$. Silva Filho et al. ${ }^{16}$ apresentam uma descrição destes sistemas de avaliação de qualidade, questionando a concordância entre os mesmos.

Huque ${ }^{17}$ define a metanálise como uma análise estatística que combina ou integra os resultados de diversos ensaios clínicos independentes, considerados "combináveis" pelo especialista. Villar et al. ${ }^{18}$ entendem a metanálise como o uso de técnicas estatísticas que combinam em uma medida resumo os resultados de estudos independentes voltados a uma única questão. As bases estatísticas da metanálise originaramse no século XVII, na astronomia, onde se estabeleceu que a combinação dos dados de diferentes estudos poderia ser mais apropriada que a observação de alguns destes trabalhos ${ }^{19}$. No século XX, o estatístico
Karl Pearson foi provavelmente o primeiro pesquisador a usar técnicas formais para combinar dados de diferentes estudos médicos, quando examinou o efeito preventivo de inoculações contra febre entérica ${ }^{20}$. Em 1976, o termo meta-analysis aparece pela primeira vez, em um artigo do psicólogo Gene Glass ${ }^{19}$, mas em um contexto filosófico, e não estatístico. Atualmente, a metanálise é uma ferramenta amplamente aceita e utilizada em várias áreas do conhecimento, como pesquisa social ${ }^{21}$, educação ${ }^{22}$ e medicina.

No campo dos ensaios clínicos controlados, um grande salto para a utilização da metanálise foi o depoimento de Archie Cochrane, médico e epidemiologista britânico, em 1979:

"Seguramente a maior crítica à nossa profissão é que nós não temos resumos críticos organizados e atualizados periodicamente, por especialidades ou subespecialidades, de todos os ensaios clínicos controlados randomizados relevantes." 23.

Cochrane elogiava as revisões sistemáticas de ensaios clínicos aleatorizados de intervenções durante a gravidez e o parto publicadas na época, sugerindo que os métodos utilizados nestes estudos deveriam ser utilizados por pesquisadores de outras especialidades ${ }^{24,25}$. Em 1992, a Colaboração Cochrane (http://www.cochrane.org) surgiu para atender a este apelo, realizando, auxiliando e disseminando revisões sistemáticas de intervenções em saúde. Em 1997 surgiu o Centro Cochrane do Brasil, seção brasileira da Colaboração Cochrane. Segundo sua home-page, é uma organização não governamental, sem fins lucrativos e sem fontes de financiamento internacionais, com a missão de elaborar, manter e divulgar revisões sistemáticas de ensaios clínicos randomizados (ver http://www.centrocochranedobrasil.org.br).

Egger e Smith ${ }^{19}$ citam que uma metanálise bem conduzida permite uma avaliação mais objetiva sobre a evidência de utilidade de um tratamento, podendo antecipar a introdução de um eficiente tratamento na prática clínica. Atallah ${ }^{25}$ afirma que uma revisão sistemática não leva menos de três meses quando conduzida por profissionais experientes, podendo ultrapassar o período de um ano. Este autor coloca ainda que é praticamente impossível uma revisão sistemática ser realizada por um único pesquisador, sendo necessários, no mínimo, dois profissionais para avaliar os ensaios clínicos. Assim, o aprendizado do trabalho em equipe, o espírito colaborativo e a capacidade de "negociar" opiniões além das evidências são ingredientes fundamentais para a qualidade da revisão sistemática ${ }^{25}$. 
Embora a metanálise seja amplamente utilizada em revisões sistemáticas de ensaios clínicos, muitos pesquisadores e estudantes de cursos da área médica ainda apresentam algumas dificuldades em compreender os métodos estatísticos envolvidos nestas análises. A diferença entre o odds ratio tradicional e o proposto por Peto, a derivação de medidas metanalíticas, o teste $Q$ de homogeneidade de Cochran e a estatística I ${ }^{2}$ ainda fomentam dúvidas a muitos principiantes em metanálises. Assim, o objetivo do presente estudo é descrever, evitando o tanto quanto possível o formalismo de expressões e derivações matemáticas, as ferramentas quantitativas mais usuais em metanálises de estudos clínicos controlados aleatorizados.

\section{2- MEDIDAS USUAIS DE TAMANHO DE EFEITO DE TRATAMENTO}

Para exemplificar o uso das medidas de tamanho de efeito de tratamento, utilizaremos como exemplo os dados da revisão sistemática publicada por Silagy et al. ${ }^{26}$, apresentados na Tabela I. Estes dados são também apresentados e discutidos por Skrondal e Rabe-Hesketh ${ }^{27}$. Trata-se de um metanálise de 27 ensaios clínicos controlados sobre o efeito da pastilha de nicotina (nicotine gum) sobre a interrupção do hábito de fumar. Em cada ensaio, os indivíduos são alocados aleatoriamente em um grupo que recebeu a pastilha de nicotina ou a um grupo que recebeu a pastilha, entretanto, sem o ingrediente ativo nicotina (um placebo). O desfecho (evento) em questão é a cessação do hábito de fumar dentro de um período de 6 meses após o tratamento.

Outras discussões sobre as medidas usuais de efeito de tratamento são apresentadas por Nobre ${ }^{28}$, Escosteguy $^{29}$ e Akobeng ${ }^{30}$.

Odds ratio: Se denotamos a probabilidade de um evento ocorrer por $p$, chamamos de odds a razão $p /(1-p)$, ou seja, quantas vezes a probabilidade deste evento ocorrer equivale à probabilidade dele não ocorrer. Por exemplo, se, em um grupo de indivíduos, a probabilidade de ocorrência de complicações após uma cirurgia é $80 \%$, temos que a probabilidade de não ocorrer complicações é $20 \%$ e o odds é $80 \% / 20 \%=4$. Isto significa que a probabilidade de ocorrer complicações após a cirurgia equivale a 4 vezes a probabilidade de não ocorrer complicações. Notar que um odds igual a 1 indica que as probabilidades de ocorrer complicações e de não ocorrer complicações são iguais. O odds ratio é o quociente entre o odds do evento em questão considerando os indivíduos submetidos ao tratamento e o odds deste mesmo evento considerando os indivíduos que compõem o grupo de controles. É, portanto, uma medida da força da associação entre o tratamento em questão e a ocorrência do evento. Considerando uma revisão sistemática que abrange $J$ estudos, o odds ratio $(O R)$ para o $j$-ésimo estudo é dado por:

$$
O R_{j}=\frac{d_{1 j} /\left(n_{1 j}-d_{1 j}\right)}{d_{0 j} /\left(n_{0 j}-d_{0 j}\right)}=\frac{d_{1 j}\left(n_{0 j}-d_{0 j}\right)}{d_{0 j}\left(n_{1 j}-d_{1 j}\right)}
$$

para $j=1,2, \ldots, J$, onde $n_{1 j}$ e $n_{2 j}$ denotam os tamanhos amostrais dos grupos de indivíduos tratados e controles do $j$-ésimo estudo, respectivamente, e $d_{1 j}$ e $d_{0 j}$ denotam o número de eventos de interesse observados nos grupos de indivíduos tratados e controles, respectivamente, do $j$-ésimo estudo. Intervalos de confiança para o odds ratio são geralmente calculados pelo método de Woolf ${ }^{31}$.

É interessante notar que, se $d_{0 j}$ é igual a zero (ou seja, o evento de interesse não ocorre entre os indivíduos do grupo de controles), o $O R$ não pode ser determinado pois sua expressão tem o valor zero em seu denominador. Isto pode ocorrer especialmente em amostras pequenas, onde o número de indivíduos não foi grande o suficiente para que ao menos um evento ocorresse. Por este motivo, o chamado odds ratio de Peto $^{32}$ é amplamente utilizado em metanálises. Esta alternativa é valida em situações onde $d_{1 j}$ é igual a zero ou $d_{0 j}$ é igual a zero.

$\mathrm{O}$ odds ratio de Peto $\left(O R_{P}\right)$ para o j-ésimo estudo é estimado por:

$$
O R_{P_{j}}=\exp \left(\frac{O_{j}-E_{j}}{V_{j}}\right)
$$

onde $O_{j}$ é igual a $d_{1 j}$, o número observado de eventos entre os indivíduos do grupo de tratados do j-ésimo estudo, $E_{j}=n_{1 j}\left(d_{1 j}+d_{0 j}\right) / n_{j}$ e $V_{j}$ é dado por:

$V_{j}=\frac{n_{1 j} n_{0 j}\left(d_{1 j}+d_{0 j}\right)\left(n_{1 j}+n_{0 j}-d_{1 j}-d_{0 j}\right)}{n_{j}^{2}\left(n_{j}-1\right)}$

considerando $n_{j}=n_{1 j}+n_{0 j}$ (ver exemplo em Altman et al. ${ }^{33}$ ). Considerando o primeiro estudo da revisão sistemática de Silagy et al. ${ }^{26}$, apresentado na Tabela I, temos: $O_{1}=37, E_{1}=92(37+24) /(92+90)=$ 
30,835 e $V_{1}=92(90)(37+24)(92+90-37-24) /$ $[1822(181)]=10,1935$. Assim, o $O R_{P 1}$ é dado por $\exp ((37-30,835) / 10,1935)=1,83$. A Tabela I mostra os odds ratio calculados pelos métodos tradicional e de Peto para os $J=27$ estudos da revisão sistemática de Silagy et al. ${ }^{26}$. Notar que, para os estudos descritos na Tabela I, $O R$ e $O R_{P}$ são bastante próximos, especialmente quando $O R$ é próximo a 1 .

Risco relativo: $\mathrm{O}$ risco relativo $(R R)$ associado ao j-ésimo estudo é dado por:

$$
R R_{j}=\frac{d_{1 j} / n_{1 j}}{d_{0 j} / n_{0 j}}=\frac{d_{1 j} n_{0 j}}{d_{0 j} n_{1 j}}
$$

para $j=1,2, \ldots, J$. Notar que, quando a incidência do evento é relativamente pequena entre os indivíduos tratados e entre os controles, o $O R$ é próximo ao $R R$. A Tabela I mostra valores de $R R$ para cada um dos estudos da revisão sistemática de Silagy et al. ${ }^{26}$. Notar que, se o pesquisador adota o $R R$ como uma medida de tamanho de efeito de tratamento, o $O R$ superestima esta medida ao apresentar uma tendência de ser maior que o $R R^{34}$.

Redução absoluta de risco: Seja $d_{0 j} / n_{0 j}$ o risco absoluto do evento de interesse entre os indivíduos controles e $d_{1 j} / n_{1 j}$ o risco do evento entre os indivíduos tratados. A redução absoluta de risco ${ }^{28}$ $(R A R)$ associada ao $j$-ésimo estudo é dada pela diferença de riscos absolutos

$$
R A R_{j}=\frac{d_{0 j}}{n_{0 j}}-\frac{d_{1 j}}{n_{1 j}}
$$

para $j=1,2, \ldots, J$. Valores positivos para $R A R$ indicam que o risco do evento é maior no grupo de indivíduos controles. Intervalos de confiança para a $R A R$ são apresentados por Anbar ${ }^{35} \mathrm{e}$ Gart e $\mathrm{Nam}^{36}$. A metanálise de estudos onde a RAR é utilizada como medida de tamanho de efeito de tratamento é discutida em Warn et al. ${ }^{37}$.

\section{3- MEDIDAS USUAIS EM METANÁLISE}

Peso de um estudo: Considerando que uma revisão sistemática envolve estudos de tamanhos amostrais diferentes, a "força" da evidência (a favor ou não do tratamento) de cada estudo não é igual.
Desta forma, convenciona-se em uma metanálise que a contribuição de cada um dos estudos à determinação de uma medida resumo deve ser relacionada ao tamanho do estudo e ao número de eventos observados. Quando o odds ratio de Peto é eleito a medida de interesse em uma metanálise, o peso de cada estudo é determinado simplesmente por $V_{j}$, calculado da forma apresentada na seção anterior. Como ilustração, seja uma metanálise envolvendo somente quatro estudos, cujos resultados fictícios são apresentados na Tabela II. Observar que, neste exemplo, o $O R$ convencional não pode ser obtido para os estudos 2 e 4 (Tabela II), dado que, em ambos, não foram observados eventos entre os indivíduos alocados no grupo de tratados. Esta é, portanto, uma situação típica onde o $O R$ de Peto é utilizado.

Na Tabela II são apresentados os valores $V_{j}$ associados a cada estudo $(j=1,2,3,4)$, calculados pela equação apresentada na seção anterior. Para facilitar a interpretação, geralmente apresenta-se em uma metanálise o peso de cada estudo em porcentagens. Estas porcentagens são dadas pelo quociente entre cada $V_{j}$ e a soma de todos os valores de $V_{j}$. Por exemplo, a contribuição relativa do estudo 1 (Tabela II) na metanálise é de 1,4808/5,232=28,3\%.

Quando utilizado o odds ratio tradicional, uma forma simples ${ }^{38}$ de determinar o peso de cada estudo é utilizando a relação $d_{0 j}\left(n_{1 j}-d_{1 j}\right) / n_{j}$, onde $n_{j}=n_{1 j}+$ $n_{0 j}$. Considerando os dados da Tabela II, o peso do estudo 1 seria $6(20-1) / 40=2,85$. O peso dos estudos 2,3 e 4 seriam, respectivamente, 1,0,3,66 e 2,5. Sendo a soma destes pesos igual a 10,01, a contribuição relativa de cada estudo à metanálise é de, respectivamente, $2,85 / 10,01=28,47 \%$ (estudo 1$)$, $1 / 10,01=9,99 \%$ (estudo 2), 36,57\% (estudo 3) e $24,97 \%$ (estudo 4). Notar que estes pesos, em porcentagens, são semelhantes àqueles encontrados pelo método de Peto (Tabela II).

É importante observar que o peso é baseado no tamanho amostral de cada estudo e no número de eventos observados, mas não diz respeito à qualidade dos estudos. O peso indica quanto cada estudo influenciará nos resultados gerais da metanálise.

Odds ratio metanalítico de Peto: O odds ratio metanalítico de Peto $\left(O R M_{P}\right)$ é dado pela função exponencial do quociente entre a soma dos valores $\mathrm{O}_{j}-\mathrm{E}_{j}$ e a soma dos valores $\mathrm{V}_{j}$. A Tabela III mostra os passos para a determinação desta medida, onde temos $O R M_{P}=\exp (-8,5915 / 5,2307)=0,1819$. 
Tabela I - Ensaios clínicos aleatorizados sobre o efeito da pastilha de nicotina (nicotine gum) sobre a interrupção do hábito de fumar (dados de Silagy et al., 2003)

\begin{tabular}{|c|c|c|c|c|c|c|c|}
\hline \multirow[b]{2}{*}{ Estudo } & \multicolumn{2}{|c|}{ Tratamento } & \multicolumn{2}{|c|}{ Controle } & \multirow[b]{2}{*}{$O R$} & \multirow[b]{2}{*}{$O R_{P}$} & \multirow[b]{2}{*}{$R R$} \\
\hline & $d_{1}$ & $n_{1}$ & $d_{0}$ & $n_{0}$ & & & \\
\hline Blondal 1989 & 37 & 92 & 24 & 90 & 1,85 & 1,83 & 1,51 \\
\hline Campbell 1991 & 21 & 107 & 21 & 105 & 0,98 & 0,98 & 0,98 \\
\hline Fagerstrom 1982 & 30 & 50 & 23 & 50 & 1,76 & 1,74 & 1,30 \\
\hline Fee 1982 & 23 & 180 & 15 & 172 & 1,53 & 1,52 & 1,47 \\
\hline Garcia 1989 & 21 & 68 & 5 & 38 & 2,95 & 2,58 & 2,35 \\
\hline Garvey 2000 & 75 & 405 & 17 & 203 & 2,49 & 2,20 & 2,21 \\
\hline Gross 1995 & 37 & 131 & 6 & 46 & 2,62 & 2,27 & 2,17 \\
\hline Hall 1985 & 18 & 41 & 10 & 36 & 2,03 & 1,99 & 1,58 \\
\hline Hall 1987 & 30 & 71 & 14 & 68 & 2,82 & 2,70 & 2,05 \\
\hline Hall 1996 & 24 & 98 & 28 & 103 & 0,87 & 0,87 & 0,90 \\
\hline Hjalmarson 1984 & 31 & 106 & 16 & 100 & 2,17 & 2,11 & 1,83 \\
\hline Huber 1988 & 31 & 54 & 11 & 60 & 6,00 & 5,28 & 3,13 \\
\hline Jarvis 1982 & 22 & 58 & 9 & 58 & 3,33 & 3,11 & 2,44 \\
\hline Jensen 1991 & 90 & 211 & 28 & 82 & 1,43 & 1,42 & 1,25 \\
\hline Killen 1984 & 16 & 44 & 6 & 20 & 1,33 & 1,32 & 1,21 \\
\hline Killen 1990 & 129 & 600 & 112 & 617 & 1,23 & 1,23 & 1,18 \\
\hline Malcolm 1980 & 6 & 73 & 3 & 121 & 3,52 & 3,64 & 3,32 \\
\hline McGovern 1992 & 51 & 146 & 40 & 127 & 1,17 & 1,17 & 1,11 \\
\hline Nakamura 1990 & 13 & 30 & 5 & 30 & 3,82 & 3,49 & 2,60 \\
\hline Niaura 1994 & 5 & 84 & 4 & 89 & 1,34 & 1,34 & 1,32 \\
\hline Niaura 1999 & 1 & 31 & 2 & 31 & 0,48 & 0,50 & 0,50 \\
\hline Pirie 1992 & 75 & 206 & 50 & 211 & 1,84 & 1,83 & 1,54 \\
\hline Puska 1979 & 29 & 116 & 21 & 113 & 1,46 & 1,45 & 1,35 \\
\hline Schneider 1985 & 9 & 30 & 6 & 30 & 1,71 & 1,69 & 1,50 \\
\hline Tonnesen 1988 & 23 & 60 & 12 & 53 & 2,12 & 2,07 & 1,69 \\
\hline Villa 1999 & 11 & 21 & 10 & 26 & 1,76 & 1,74 & 1,36 \\
\hline Zelman 1992 & 23 & 58 & 18 & 58 & 1,46 & 1,45 & 1,28 \\
\hline
\end{tabular}


Tabela II - Dados fictícios de uma metanálise envolvendo quatro estudos, exemplificando a determinação do peso de cada estudo

\begin{tabular}{|c|c|c|c|c|c|c|c|c|}
\hline \multirow[b]{2}{*}{ Estudo } & \multicolumn{2}{|c|}{ Tratamento } & \multicolumn{2}{|c|}{ Controle } & \multirow{2}{*}{$\begin{array}{c}\text { OR } \\
\text { de Peto }\end{array}$} & \multirow[b]{2}{*}{ IC $95 \%$} & \multirow[b]{2}{*}{$V$} & \multirow{2}{*}{$\begin{array}{r}\text { Peso } \\
(\%)\end{array}$} \\
\hline & $d_{1}$ & $n_{1}$ & $d_{0}$ & $n_{0}$ & & & & \\
\hline 1 & 1 & 20 & 6 & 20 & 0,185 & $(0,037 ; 0,925)$ & 1,4808 & 28,30 \\
\hline 2 & 0 & 15 & 2 & 15 & 0,126 & $(0,008 ; 2,116)$ & 0,4828 & 9,23 \\
\hline 3 & 2 & 29 & 8 & 30 & 0,251 & $(0,065 ; 0,969)$ & 2,1115 & 40,36 \\
\hline 4 & 0 & 27 & 5 & 27 & 0,120 & $(0,019 ; 0,741)$ & 1,1570 & 22,11 \\
\hline Soma & & 91 & & 92 & & & 5,2320 & 100,00 \\
\hline
\end{tabular}

Tabela III - Dados fictícios de uma metanálise envolvendo quatro estudos, exemplificando a determinação do OR de Peto metanalítico

\begin{tabular}{|c|c|c|c|c|c|c|c|c|c|}
\hline \multirow[b]{2}{*}{ Estudo } & \multicolumn{2}{|c|}{ Tratamento } & \multicolumn{2}{|c|}{ Controle } & \multirow{2}{*}{$\begin{array}{c}O R \\
\text { de Peto }\end{array}$} & \multirow[b]{2}{*}{$\mathrm{O}$} & \multirow[b]{2}{*}{$\mathrm{E}$} & \multirow[b]{2}{*}{$\mathrm{O}-\mathrm{E}$} & \multirow[b]{2}{*}{ V } \\
\hline & $d_{1}$ & $n_{1}$ & $d_{0}$ & $n_{0}$ & & & & & \\
\hline 1 & 1 & 20 & 6 & 20 & 0,185 & 1 & 3,500 & $-2,500$ & 1,4808 \\
\hline 2 & 0 & 15 & 2 & 15 & 0,126 & 0 & 1,000 & $-1,000$ & 0,4828 \\
\hline 3 & 2 & 29 & 8 & 30 & 0,251 & 2 & 4,915 & $-2,915$ & 2,1115 \\
\hline 4 & 0 & 27 & 5 & 27 & 0,120 & 0 & 2,500 & $-2,500$ & 1,1557 \\
\hline Soma & & 91 & & 92 & & & & $-8,915$ & 5,2307 \\
\hline
\end{tabular}

Greenland e Salvan ${ }^{39}$ advertem que o $O R M_{P}$ é sujeito a vieses quando a metanálise inclui estudos não balanceados quanto ao número de indivíduos que compõem os grupos de tratamento e controle.

Odds ratio metanalítico de MantelHaenszel: Quando utilizados odds ratios calculados pela maneira tradicional, o odds ratio metanalítico pode ser determinado pelo método de Mantel e Haenszel $^{40}$. O odds ratio metanalítico de Mantel e Haenszel $\left(O R M_{M H}\right)$ é uma média entre os odds ratios encontrados em cada estudo $\left(O R_{j}\right)$, ponderada pelos respectivos pesos dados por $d_{0 j}\left(n_{1 j}-d_{1 j}\right) / n_{j}$. Considerando os dados da Tabela I, temos que o peso do primeiro estudo é $24(92-37) / 182=7,25$. O peso do segundo estudo é $21(107-21) / 212=8,52$, e assim por diante. O $O R M_{M H}$ é dado pela soma dos produtos entre cada $O R_{j}$ e o seu respectivo peso, dividida pela soma entre todos os pesos, ou seja,

$$
O R M_{M H}=\frac{\text { somd } \left.\text { peso }_{j} \times O R_{j}\right)}{\text { somd })}
$$

Assim, na revisão sistemática publicada por Silagy et al. ${ }^{26}$, temos $O R M_{M H}=1,66$. Intervalos de confiança para $O R M_{M H}$ são apresentados por Robins et al. ${ }^{41}$. Neste exemplo, temos um intervalo de confiança $95 \%$ igual a $(1,46 ; 1,89)$.

É importante destacar que $O R M_{P}$ e $O R M_{M H}$ são medidas que pressupõem que os estudos considerados são homogêneos, ou seja, a variabilidade entre os 
estudos é casual, e não devida às possíveis diferenças entre as populações, intervenções e protocolos empregados. A diversidade entre os estudos é um aspecto fundamental na metanálise, pois quanto mais homogêneos forem os resultados a serem combinados, mais confiável será a estimativa combinada.

Teste $Q$ de homogeneidade de Cochran: Cochran ${ }^{42}$ introduziu um teste de hipóteses para a homogeneidade entre diferentes experimentos (não confundir com o conhecido teste de Cochran para amostras pareadas, descrito em Fleiss et al. ${ }^{43}$ (p.389)). Este teste é baseado na quantidade $Q$ que, considerando os dados da Tabela II, é dada por:

$$
Q=\operatorname{som} a\left[\frac{\left(\mathrm{O}_{j}-\mathrm{E}_{j}\right)^{2}}{V_{j}}\right]-\frac{\left[\operatorname{soma}\left(\mathrm{O}_{j}-\mathrm{E}_{j}\right)\right]^{2}}{\operatorname{soma}_{j}}=15,725-\frac{(-8,915)^{2}}{5,2307}=0,53 \text {, }
$$
tatisticamente significante", porém, clinicamente não importante. Perante estas desvantagens, Higgins e Thompson ${ }^{45}$ propuseram a estatística $\mathrm{I}^{2}$ com o propósito de quantificar a heterogeneidade entre os estudos envolvidos em uma metanálise.

quando utilizado o $O R$ de Peto $\left(O R_{P}\right)$ como medida de tamanho de efeito. Considerando uma metanálise que abrange $J$ estudos e sob a hipótese que estes estudos são homogêneos, $Q$ segue uma distribuição qui-quadrado com $J-1$ graus de liberdade (gl). Assim, $p$ valores são obtidos comparando o valor de $Q$ encontrado na metanálise com uma distribuição quiquadrado. Considerando $J-1=3 \mathrm{gl}$, temos um $p$ valor igual a 0,91 (este $p$ valor pode ser facilmente obtido no programa Microsoft Excel digitando em uma célula a expressão $=$ DIST.QUI $(0,53 ; 3))$. Como o $p$ valor é grande, temos evidências de que os estudos são homogêneos.

Ainda considerando os dados da Tabela II, por ilustração, vamos considerar agora que, no estudo 3 , $d_{1}=5$ e $d_{0}=2$. Note que, com esta mudança, o $O R_{P}$ do terceiro estudo é bastante diferente dos demais $\left(\mathrm{OR}_{3}=2,702\right)$, evidenciando uma maior heterogeneidade entre os estudos. Temos agora $O R M_{P}=0,388$, ou seja, o $O R$ metanalítico é "inflacionado" pelos resultados do estudo 3. A estatística $Q$ do teste de Cochran é dada por $Q=9,04$, e o respectivo $p$ valor é 0,03 . Desta vez, um $p$ valor pequeno (menor que os tradicionais 5\%) evidencia que os estudos envolvidos na metanálise são heterogêneos.

Quando utilizado o odds ratio tradicional, uma maneira de obter a estatística $Q$ é pela soma dos quadrados das diferenças entre o logaritmo do $O R$ de cada estudo e o logaritmo de $O R M_{M H}$, sendo que cada termo desta soma é multiplicado pelo peso do estudo. Considerando os dados da Tabela I, temos $Q=26,2$ $(p=0,45)$.
Estatística $\mathbf{I}^{\mathbf{2}}$ : A estatística $\mathrm{I}^{2}$, interpretada como a porcentagem total de variação entre os estudos envolvidos em uma metanálise devida a heterogeneidade entre estes, é obtida da equação:

$$
\mathrm{I}^{2}=100 \%(Q-J+1) / Q,
$$

onde $Q$ é a estatística do teste de Cochran e $J$ é o número de estudos contidos na metanálise. A estatística $\mathrm{I}^{2}$ varia de $0 \%$ a $100 \%$, sendo que valores negativos são considerados iguais a zero ${ }^{44}$. Um valor igual a $0 \%$ evidencia homogeneidade entre os estudos, e valores grandes traduzem a magnitude da heterogeneidade. Uma alta heterogeneidade é interpretada como um indicativo de inconsistência entre os resultados dos estudos.

Considerando os dados da Tabela II, temos $\mathrm{I}^{2}=$ $100 \%(0,53-3) / 0,53=-4,66 \%$. Como I ${ }^{2}$ é, neste caso, negativo, consideramos $\mathrm{I}^{2}=0 \%$ e interpretamos este resultado como uma evidência de que os estudos são homogêneos.

Enquanto Higgins et al. ${ }^{44}$ mostram que uma desvantagem do teste de Cochran é que seu poder depende do número de estudos, a estatística $\mathrm{I}^{2}$ é corrigida pela quantidade de estudos considerados na metanálise. Intervalos de confiança para $\mathrm{I}^{2}$ são apresentados em Higgins e Thompson ${ }^{45}$.

\section{4- REPRESENTAÇÃO GRÁFICA}

Os resultados de uma metanálise são geralmente apresentados em uma representação gráfica cha- 
mada forest plot. Lewis e Clarke ${ }^{46}$ mencionam que as origens deste nome são obscuras, e há a sugestão de que o gráfico é assim chamado por possuir a aparência de uma "floresta de linhas". A Figura 1 traz um exemplo de um forest plot para os dados da Tabela II. Na coluna à esquerda da figura, são listados os estudos utilizados na metanálise. A segunda coluna contém os dados do grupo de tratamentos de cada estudo. Seus valores indicam o número de eventos $\left(d_{1}\right)$ e o tamanho amostral do grupo $\left(n_{1}\right)$. A coluna seguinte traz os dados do grupo de controles (respectivamente $d_{0}$ e $n_{0}$ ). As linhas horizontais representam intervalos de confiança $95 \%$ para o odds ratio de Peto estimado em cada estudo. Observar que tamanhos amostrais relativamente pequenos trazem menor precisão, e, conseqüentemente, intervalos de confiança com maior amplitude.

Os pontos sobre cada linha horizontal representam o odds ratio de Peto de cada respectivo estudo, sendo que o tamanho destes pontos é diretamente proporcional ao respectivo peso (exibidos na primeira coluna à direita da figura). Notar que os pontos não são representados necessariamente no centro das linhas, dado que os intervalos de confiança para o $O R$ não são simétricos em relação a esta medida. A linha vertical pontilhada mostra onde seriam representados odds ratios iguais a 1, que indicariam a ausência de uma associação entre o tratamento em estudo e a ocorrência do evento considerado. Assim, odds ratios representados à esquerda desta linha vertical evidenciam que o evento é mais propenso a ocorrer dentre os controles; e odds ratios representados à direita desta linha evidenciam que o evento tem maior propensão a ocorrer dentre os tratados.

Se a linha horizontal atravessa a linha pontilhada, o intervalo de confiança $95 \%$ contém então o valor 1 , o que traz evidências de que naquele respectivo estudo o efeito do tratamento sobre a ocorrência do evento é não significativo (a interpretação é análoga à usualmente feita quando o $p$ valor é maior que 5\%, mas lembrar que este conceito de "significância estatística" é sempre influenciado pelo tamanho amostral). $\mathrm{Na}$ Figura 1, notamos que o intervalo de confiança associado ao estudo 2 contém o valor 1, indicando que o seu respectivo OR é "não significativo". Por outro lado, notar que o estudo 2 possui um tamanho amostral menor que os demais, sugerindo a possibilidade desta "significância" não ter sido atingida pelo número reduzido de participantes no estudo. Esta discussão leva ao conceito do "poder" do teste de significância, que não será aqui apresentado por demandar uma discussão bastante ampla.

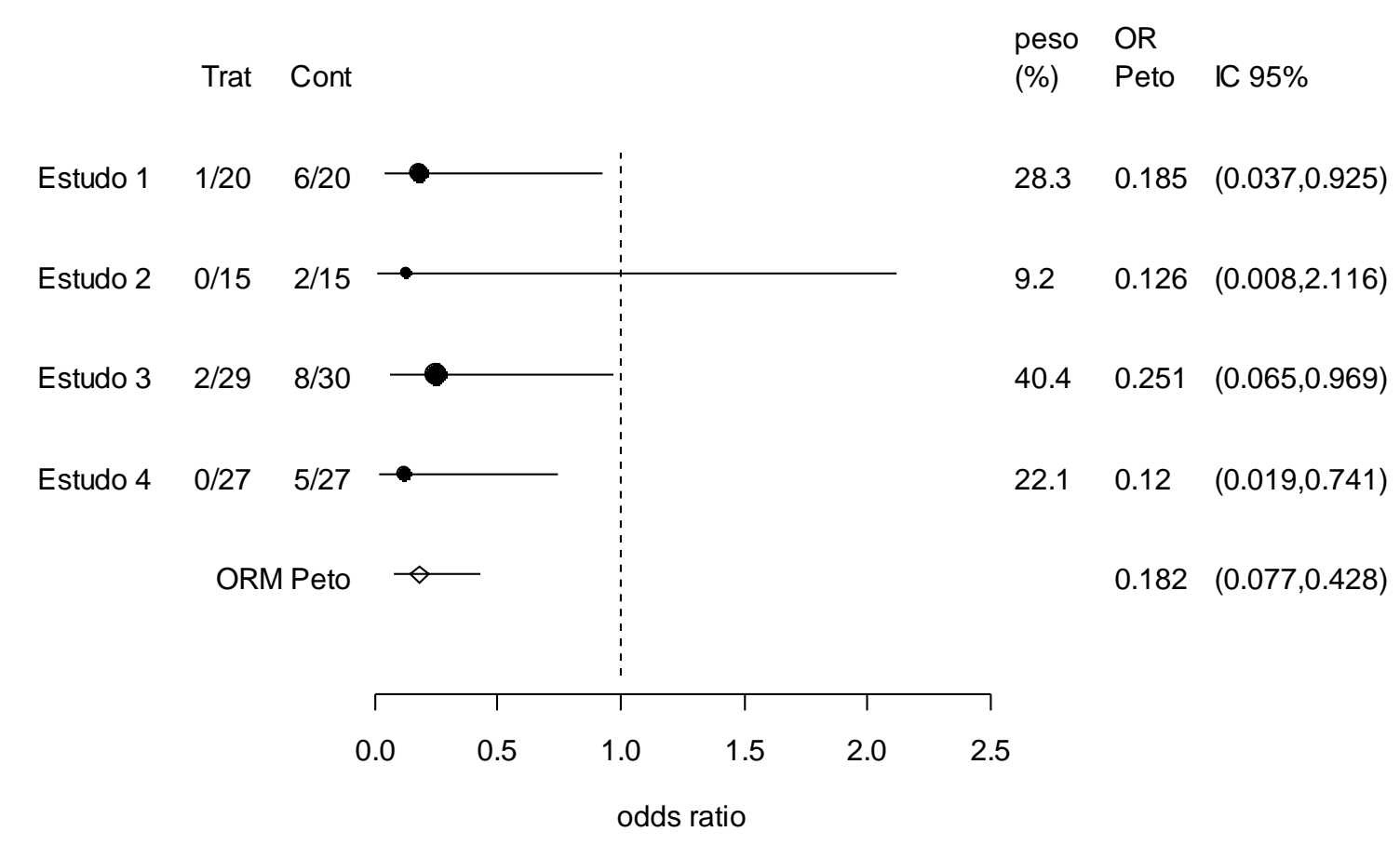

Figura 1 - Forest plot para dados fictícios de uma metanálise envolvendo quatro estudos, onde $Q=0,53(p=0,91)$ e $\mathrm{I}^{2}=0 \%$. 
Finalmente, o $O R$ metanalítico é representado abaixo do conjunto de linhas horizontais, com seu respectivo intervalo de confiança (este também representado por uma linha horizontal). Costuma-se representar o $O R$ metanalítico por um losango, como na Figura 1, para diferenciá-lo dos odds ratios associados a cada um dos estudos. Na Figura 1, nota-se que o intervalo de confiança para o $O R$ metanalítico tem naturalmente uma amplitude menor que a dos intervalos de confiança individuais, dado que ele reúne informações de todos os estudos considerados.

A Figura 2 mostra o forest plot para a metanálise do efeito da pastilha de nicotina sobre a interrupção do hábito de fumar (Tabela I). Os odds ratios e seus intervalos de confiança são agora representados em uma escala logarítmica, o que é mais comum na apresentação de resultados de metanálises. Notar que os intervalos de confiança, representados em escala logarítmica, são agora simétricos em relação ao $O R$ (os odds ratios agora aparecem exatamente no centro das linhas horizontais). Neste gráfico, a maioria dos odds ratios aparece à direita da linha vertical pontilhada que atravessa a escala em zero (lembrando que estamos considerando uma escala logarítmica, e o logaritmo de 1 é zero), evidenciando que a interrupção do hábito de fumar é mais incidente entre os indivíduos que receberam a pastilha de nicotina. $\mathrm{O}$ odds ratio metanalítico de Mantel-Haenszel traz um intervalo de confiança que não atravessa a linha vertical, o que permite concluir que há um "significativo" efeito do tratamento sobre a interrupção do hábito de fumar.

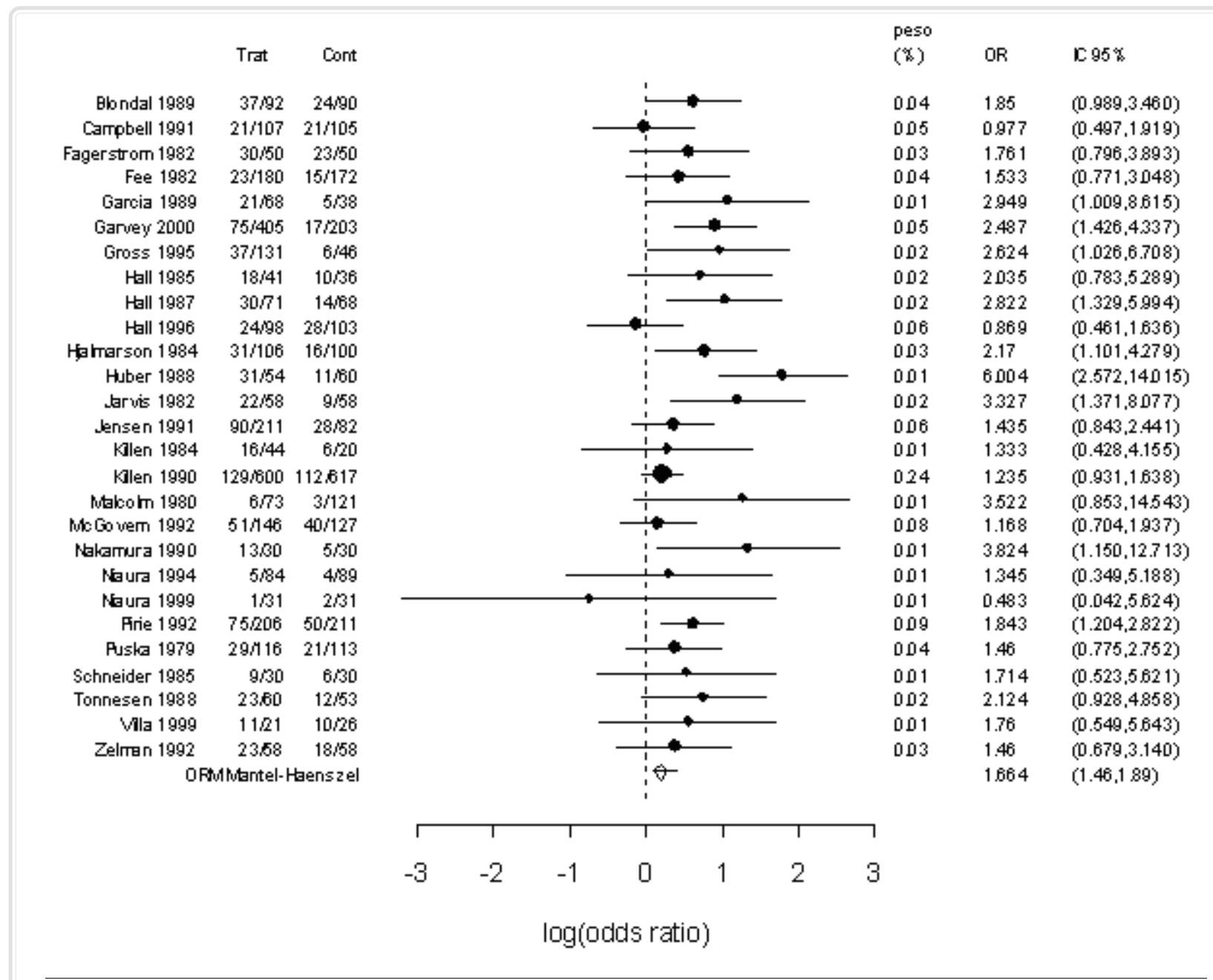

Figura 2: Forest plot para a metanálise do efeito da pastilha de nicotina (nicotine gum) sobre a interrupção do hábito de fumar (dados de Silagy et al, 2003). 


\section{5- MODELOS DE REGRESSÃO EM META- NÁLISE}

O desenvolvimento de modelos de regressão voltados à metanálise tem despertado o interesse de muitos estatísticos ${ }^{18,47}$. Há essencialmente dois tipos de regressão em metanálise: os modelos de efeitos fixos e os modelos de efeitos aleatórios. Os modelos de efeitos fixos assumem a existência de um único efeito de tratamento e qualquer variabilidade entre os efeitos estimados de tratamentos dos estudos é completamente devida à variabilidade amostral interna de cada um dos estudos ${ }^{27}$. Este pressuposto de homogeneidade entre os estudos é geralmente testado pelo teste $Q$ de Cochran. Os modelos de efeitos aleatórios, por sua vez, assumem que os estudos não são homogêneos, havendo alguma variação entre os estudos devida a diferenças entre as respectivas populações e protocolos empregados (por exemplo, dosagens ou duração do tratamento).

Quando a medida de tamanho de efeito de tratamento utilizada na metanálise é o odds ratio, os modelos de regressão buscam analisar o logaritmo do odds ratio $(\ln (O R))$ devido à sua melhor aproximação a uma distribuição normal. $\mathrm{O}$ modelo de efeitos aleatórios possui a forma forma $\ln \left(O R_{j}\right)=\beta+\zeta_{j}+$ $\varepsilon_{j}$, onde $\beta$ é o odds ratio metanalítico, $\zeta_{j}$ é um efeito aleatório, representando o desvio do odds ratio do j-ésimo estudo em relação ao odds ratio metanalítico, e $\varepsilon_{j}$ é o resíduo do modelo, com distribuição normal com média 0 e variância $\sigma_{j}^{2}$ dada pelo método de Woolf ${ }^{31}$ :

$$
\sigma_{j}^{2}=\frac{1}{d_{0 j}}+\frac{1}{n_{0 j}-d_{0 j}}+\frac{1}{d_{1 j}}+\frac{1}{n_{1 j}-d_{1 j}}
$$

considerando o odds ratio calculado pelo modo tradicional. Notar que uma característica deste modelo é que não se assume que a variância do resíduo é constante, como é tradicionalmente feito nos modelos de regressão, mas admite-se que cada estudo contribua com uma medida $\sigma_{j}^{2}$. O modelo de efeitos fixos possui a mesma forma, exceto pela ausência de $\zeta_{j}$, ou seja, $\ln \left(O R_{j}\right)=\beta+\varepsilon_{j}$,

Utilizando o programa SAS versão 9 e considerando os estudos descritos na Tabela I, o parâmetro $\beta$ do modelo de regressão de efeitos fixos $\ln \left(O R_{j}\right)=\beta$ $+\varepsilon_{j}$, é estimado em 0,4983 (com erro padrão 0,06634). $\mathrm{O}$ odds ratio metanalítico estimado por este modelo é, portanto, $\mathrm{e}^{\beta}=1,65$, com intervalo de confiança $95 \%$ dado por $(1,43 ; 1,89)$. Notar que o $O R$ encontrado pelo modelo de regressão é bastante próximo ao
$O R M_{M H}$, estimado em 1,664 com um intervalo de confiança 95\% $(1,46 ; 1,89)$ (ver Figura 2).

$\mathrm{O}$ modelo de regressão de efeitos aleatórios $\ln \left(O R_{j}\right)=\beta+\zeta_{j}+\varepsilon_{j}$, por sua vez, estima $\beta \operatorname{em} 0,5583$. Assim, não considerando homogeneidade entre os estudos, o odds ratio metanalítico é dado por $\mathrm{e}^{\beta}=1,75$ com intervalo de confiança $95 \%(1,46 ; 2,09)$. Notar que, não considerando o pressuposto de homogeneidade, o $O R$ é corrigido pelo efeito aleatório $\zeta_{j}$ introduzido no modelo de regressão.

Modelos de regressão para metanálises sob um contexto Bayesiano são apresentados por Sutton e Abrams ${ }^{48}$ e Warn et al. ${ }^{37}$.

\section{6- RECURSOS COMPUTACIONAIS}

Alguns programas comerciais de computador trazem grandes facilidades ao pesquisador que está desenvolvendo uma metanálise. O programa Comprehensive Meta-Analysis (http://www.MetaAnalysis.com) traz diversos recursos, incluindo gráficos e ajustes de modelos de regressão. O Cochrane Collaboration's Review Manager software (RevMan ${ }^{49}$; http://www.cc-ims.net/RevMan) e o NCSS (http://www.ncss.com/metaanal. html) também trazem bons recursos de metanálise.

Sheu e Suzuki ${ }^{50}$ trazem um roteiro para o uso dos modelos de regressão de efeitos aleatórios com o procedimento PROC MIXED do programa SAS. Houwelingen et al. ${ }^{46}$ apresentam outros exemplos do uso do programa SAS em metanálise. O ajuste de modelos de regressão sob um enfoque Bayesiano, considerando efeitos fixos ou aleatórios, é facilitado pelo programa Winbugs (disponível gratuitamente em http://www.mrc-bsu.cam.ac.uk/bugs/). O primeiro volume do manual de exemplos do Winbugs ${ }^{51}$ traz uma ilustração do uso deste programa no ajuste de modelos de regressão em metanálises.

\section{7- COMENTÁRIOS FINAIS}

Perante uma questão clínica relevante sobre intervenções terapêuticas onde não existe um consenso dos estudos publicados, ou quando não há uma comprovação adequada da eficácia de um procedimento, a metanálise pode trazer importantes informações para as decisões sobre condutas, desde que alguns critérios sejam atendidos. Estes critérios incluem uma questão clínica adequadamente formulada, uma busca completa e abrangente dos estudos em fontes confiáveis, a garantia da qualidade metodológica dos estudos e a 
utilização de ferramentas estatísticas adequadas. Embora programas de computador estejam atualmente disponíveis para a condução de metanálises, o uso destes programas como "caixas-pretas", sem o conhecimento adequado dos métodos estatísticos neles implementados, pode trazer falsas interpretações sobre a variabilidade dos estudos e sobre as medidas usuais da metanálise. O presente artigo busca suprir especificamente este critério.

Embora a metanálise seja uma ferramenta analítica aceita em diversas áreas do conhecimento, constante em muitos artigos publicados nos mais conceituados periódicos indexados no Medline, não deixa de receber a desconfiança de pesquisadores que clamam por uma medicina baseada em evidências objetivas. Como exemplo, Voltarelli ${ }^{52}$ discute a descrença de pesquisadores médicos em metanálises, mencionando uma frase de H.J.Eysenck, da Universidade de Londres: "If a medical treatment has an effect so recondite and obscure to require meta-analysis to establish it, I would not be happy to have it used on me. It would seem better to improve the treatment, and the theory underlying the treatment".

Decisões de condutas terapêuticas e preventivas dependem de informações científicas que devem com imprescindibilidade possuir confiabilidade e precisão. O discernimento sobre o nível de evidência de um achado de investigação é, portanto, essencial para julgar o mérito deste achado nesta tomada de decisões. Às opiniões de especialistas pode ser atribuído um nível pequeno, porém importante, de evidência. Em uma ordem crescente, podemos atribuir maiores níveis de evidência ${ }^{53,54,55}$ às séries de casos, aos estudos caso-controle, e aos estudos de coorte ${ }^{56,57}$. Em seguida, aparecem os ensaios clínicos aleatorizados. Primeiro, os ensaios pequenos, com menos de 1000 pacientes, e, portadores de um nível maior de evidência, os megatrials com mais de 1000 pacientes. As revisões sistemáticas de ensaios clínicos, com metanálise e homogeneidade entre os estudos, apareceriam como o processo de investigação científica merecedor de maior nível de evidência. No entanto, as controvérsias sobre a metanálise são prevalentes ${ }^{19}$, sendo que alguns pesquisadores acreditam que misturar resultados de estudos traz conclusões inadequadas ${ }^{58}$, portanto perigosas para a tomada de decisões. Outra crítica importante é quanto à sua exposição ao viés de seleção ${ }^{59}$, conseqüente do fato de estudos com resultados favoráveis a uma intervenção possuírem maiores chances de serem publicados e incluídos na metanálise.

Martinez EZ. Meta-analysis of randomized controlled trials: quantitative aspects. Medicina (Ribeirão Preto) 2007; 40 (2): 223-35.

ABSTRACT: A meta-analysis combines the results of several studies that address a set of related research hypotheses. Meta-analyses has been taking place in the medical literature, specialy in systematic reviews of randomized control trials. Therefore, there is a need for more works on the underlying statistical methodology. Results from studies are combined using different statistical approaches. One approach frequently used in meta-analysis of randomized controlled trials considers that there is homogeneity across all studies. Regression models with fixed effects are compatible with this assumption. Other approach considers heterogeneity across the studies. Random effects regression models are used in this situation. The present article reviews some quantitative aspects of the meta-analysis.

Keywords: Meta-Analysis. Clinical Trials. Systematic Review. Statistics.

\section{REFERÊNCIAS}

1 - Pocock S. Clinical trials: a practical approach. Chinchester: John Wiley \& Sons; 1983.

2 - Boissel JP. Planning of clinical trials. J Intern Med 2004; 255: 427-38.
3 - Kendall JM. Designing a research project: randomised controlled trials and their principles. Emerg Med J 2003; 20:164-8

4 - Fisher RA. The design of experiments. Edinburg: Oliver and Boyd, 1935.

5 - Altman DG, Bland JM. Treatment allocation in controlled trials: why randomise? BMJ 1999; 318:1209. 
6 - Roberts C, Torgerson D. Understanding controlled trials: Randomisation methods in controlled trials. BMJ 1998; 317:1301-10.

7 - Torgerson DJ, Roberts C. Understanding controlled trials: Randomisation methods: concealment. BMJ 1999; 319: 375-6.

8 - Altman DG, Bland JM. Statistics notes: How to randomise. BMJ 1999; 319:703-4.

9 - Atallah AN. Systematic reviews of medical literature and metanalyses. Sao Paulo Med J 1996; 114:1285-7.

10 - Akobeng AK. Understanding systematic reviews and metaanalysis. Arch Dis Child 2005; 90:845-8.

11 - Mulrow CD. Systematic reviews: rationale for systematic reviews. BMJ 1994; 309:597-9.

12 - Haines SJ, Walters BC. What is metanalysis? Surg Neurol 1995; 44:581-2.

13 - Verhagen AP, de Vet HC, de Bie RA, Kessels AG, Boers M, Knipschild PG. Balneotherapy and quality assessment: interobserver reliability of the Maastricht criteria list and the need for blinded quality assessment. J Clin Epidemiol 1998; 51: 335-41.

14 - Jadad AR, Moore RA, Carroll D, Jenkinson C, Reynolds DJ, Gavaghan DJ, et al. Assessing the quality of reports of randomized clinical trials: is blinding necessary? Control Clin Trials 1996;17:1-12.

15 - Verhagen AP, Vet HC, Bie RA, Kessels AG, Boers M, Bouter LM, et al. The Delphi list: a criteria list for quality assessment of randomized clinical for conducting sistematic reviews developed by Delphi consensus. J Clin Epidemiol 1998; 51:1235-41.

16 - Silva Filho CR, Saconato H, Conterno LA, Marques I, Atallah AN. Avaliação da qualidade de estudos clínicos e seu impacto nas metanálises. Rev Saude Publica 2005; 39: 86573.

17 - Huque MF. Experiences with meta-analysis in NDA submissions. Proceedings of the Biopharmaceutical Section of the American Statistical Association 1988; 2:28-33.

18 - Villar J, Mackey ME, Carroli G, Donner A. Meta-analyses in systematic reviews of randomized controlled trials in perinatal medicine: comparison of fixed and random effects models. Stat Med. 2001; 20:3635-47.

19 - Egger M, Smith GD. Meta-analysis: potentials and promise. BMJ 1997; 315:1371-4.

20 - Pearson K. Report on certain enteric fever inoculation statistics. BMJ 1904; 3:1243-6.

21 - Glass GV, McGaw B, Smith ML. Meta-analysis in social research. Beverly Hills (CA): Sage; 1981.

22 - Kulik JA, Kulik CLC. Meta-analysis in education. Int J Educ Res 1989; 13: 221-340.

23 - Cochrane AL. 1931-1971: a critical review, with particular reference to the medical profession. In: Medicines for the year 2000. London: Office of Health Economics, 1979, p. 1-11.

24 - Cochrane AL. Foreword. In: Chalmers I, Enkin M, Keirse MJNC, editors. Effective care in pregnancy and childbirth. Oxford: Oxford University Press; 1989.
25 - Atallah AN. Revisão sistemática da literatura e metanálise. In: Atallah AN, Castro AA, editores. Medicina baseada em evidências: fundamentos da pesquisa clínica. São Paulo: Lemos-Editorial; 1998. p.42-8.

26 - Silagy C. Nicotine replacement therapy for smoking cessation (Cochrane review). In: The Cochrane Library Issue 4. Chichester: Wiley, 2003.

27 - Skrondal A, Rabe-Hesketh S. Generalized latent variable modeling: multilevel, longitudinal and structural equation models. Boca Raton:Chapman \& Hall/CRC, 2004.

28 - Nobre MRC. Não compre gato por lebre - olhe o risco absoluto e o intervalo de confiança antes de uma decisão clínica. Rev Bras Reumatol 2001; 41:247.

29 - Escosteguy CC. Estudos de intervenção. In: Medronho RA, editor. Epidemiologia. São Paulo: Editora Atheneu; 2002. p.151-160.

30 - Akobeng AK. Understanding measures of treatment effect in clinical trials. Arch Dis Child 2005; 90:54-6.

31 - Woolf B. On estimating the relationship between blood groups and disease. Ann Hum Genet 1955; 19: 251-3.

32 - Yusuf S, Peto R, Lewis J, Collins R, Sleight P. Beta blockade during and after myocardial infarction: an overview of the randomized trials. Prog Cardiovasc Dis 1985, 27: 335-71.

33 - Altman R, Carreras L, Diaz R, Figueroa E, Paolasso E, Parodi $\mathrm{JC}$, et al. Collaborative overview of randomized trials of antiplatelet therapy. I. Prevention of death, myocardial infarction and stroke by prolonged antiplatelet therapy in various categories of patients. BMJ 1994; 308:81-106.

34 - Rigby AS. Statistical methods in epidemiology III. The odds ratio as an approximation to the relative risk. Disabil Rehabil 1999; 21: 145-51.

35 - Anbar D. On estimating the difference between two probabilities, with special reference to clinical trials. Biometrics 1983; 39:257-62.

36 - Gart JJ, Nam J. Approximate interval estimation for the difference in binomial parameters: correction for skewness and extension to multiple tables. Biometrics 1990; 46:637-43.

37 - Warn DE, Thompson SG, Spiegelhalter DJ. Bayesian random effects meta-analysis of trials with binary outcomes: methods for the absolute risk difference and relative risk scales. Stat Med 2002; 21:1601-23.

38 - Petitti DB. Meta-analysis, decision analysis, and cost-effectiveness analysis. New York: Oxford University Press; 1994.

39 - Greenland S, Salvan A. Bias in the one-step method for pooling study results. Stat Med 1990; 9:247-52.

40 - Mantel N, Haenszel W. Statistical aspects of the analysis of data from retrospective studies of disease. J Natl Cancer Inst 1959; 22:719-48.

41 - Robins J, Greenland S, Breslow NE. A general estimator for the variance of the Mantel-Haenszel odds ratio. Am J Epidemiol 1986;124:719-23.

42 - Cochran WG. The combination of estimates from different experiments. Biometrics 1954; 10:101-29.

43 - Fleiss JL, Levin B, Paik MC. Statistical methods for rates and proportions. 3rd.ed. New Jersey: John Wiley \& Sons, 2003. 
44 - Higgins JPT, Thompson SG, Deeks JJ, Altman DG. Measuring inconsistency in meta-analyses. BMJ 2003; 327: 557-60.

45 - Higgins JPT, Thompson SG. Quantifying heterogeneity in a metaanalysis. Stat Med 2002; 21: 1539-58.

46 - Lewis S, Clarke M. Forest plots: trying to see the wood and the trees. BMJ 2001; 322:1479-80.

47 - Houwelingen HCV, Arends LR, Stijnen T. Advanced methods in meta-analysis: multivariate approach and meta-regression. Stat Med 2002; 21:589-624.

48 - Sutton AJ, Abrams KR. Bayesian methods in meta-analysis and evidence synthesis. Stat Methods Med Res 2001; 10: 277-303.

49 - Review Manager (RevMan) [Computer program]. Version 4.2 for Windows. Oxford, England: The Cochrane Collaboration; 2002.

50 - Sheu CF, Suzuki S. Meta-analysis using linear mixed models. Behav Res Methods Instrum Comput 2001; 33: 102-7.

51 - Spiegelhalter DJ, Thomas A, Best NG, Gilks WR. BUGS 0.5 Examples, Volume 1. Cambridge: MRC-Biostatistics Unit; 1996.

52 - Voltarelli JC. Editorial: anistia a ensaios clínicos. Medicina, Ribeirão Preto 1998; 31: 1-2.

53 - Atallah AN. Tomada de decisões em terapêutica. Diagn Tratamento $2001 ; 6: 46$.
54 - Atallah AN. Tomada de decisões em terapêutica. Diagn Tratamento $2001 ; 6: 54$.

55 - Atallah AN. Princípios metodológicos para tomada de decisões médicas. Diagn Tratamento 2001; 6:47.

56 - Avezum A. Cardiologia Baseada em Evidências - IV. Principais Estratégias de Pesquisa e Níveis de Recomendações em Cardiologia. Arq Bras Cardiol 1998; 71: 649-52.

57 - Medeiros LR, Stein A. Níveis de evidência e graus de recomendação da medicina baseada em evidências. Rev AMRIGS Porto Alegre 2002, 46: 43-6.

58 - Feinstein AR. Meta-analysis: statistical alchemy for the 21st century. J Clin Epidemiol 1995; 41: 71-9.

59 - Sterne JAC, Gavaghan D, Egger M. Publication and related bias in meta-analysis: Power of statistical tests and prevalence in the literature. J Clin Epidemiol 2000; 53: 1119-29.

Recebido em 04/09/2006

Aprovado em 11/04/2007 